\title{
THEORY
}

\section{Twenty-First-Century Spaces for Twenty-First-Century Learners: Where We Are, How We Got Here, and What Next}

\author{
Jeanne L. Narum, Learning Spaces Collaboratory \\ Kimberley Frederick, Skidmore College \\ Michael A. Palladino, Monmouth University
}

\begin{abstract}
Appropriately designed laboratory and classroom facilities are essential for facilitating high-quality original research experiences for undergraduate students. Project Kaleidoscope (PKAL) — an initiative funded by the National Science Foundation (NSF) that involved leaders of the Council on Undergraduate Research (CUR)—engaged faculty, administrators, architects, campus planners, and other key stakeholders in the intentional design of learning spaces for undergraduate research at primarily undergraduate institutions (PUIs). This article discusses nearly three decades of experience since the initiation of the movement to improve STEM facilities at PUIs nationwide. It poses questions for consideration at the beginning of a space-planning process and presents examples of planning processes from Monmouth University and Skidmore College. It also discusses the Learning Spaces Collaboratory (LSC) and a roundtable model for learner-centered facility planning based on lessons learned.
\end{abstract}

Keywords: learning spaces, Learning Spaces Collaboratory, Project Kaleidoscope, science facilities, space planning

doi: 10.18833/spur/1/2/11

\section{Background}

In the mid-1980s, there was growing concern about the decreasing numbers of undergraduates who intended to pursue doctoral studies and become scientists. The problem was widely recognized but had not yet catalyzed action on campuses or at the National Science Foundation (NSF). In 1986, the National Science Board issued a report (NSB 1986) to NSF that clarified the problem and expressed a national urgency that it be addressed. The report's authors called on NSF to gather the sense of the community as a prelude to considering new policies, programs, and budgets in support of undergraduate initiatives at NSF. In 1989, NSF invited Jeanne L. Narum to convene a working group representing primarily undergraduate institutions (PUIs) to draft recommendations for the future of undergraduate programs in disciplines funded by NSF. Funding an effort that became Project Kaleidoscope (PKAL) was one step toward gaining that "sense of the community." PKAL's charge from NSF was clear from the start: "Don't point the finger; point the way." Fortunately for PKAL, evidence was starting to emerge about the best measures for achieving success on what works. Data and stories from the pioneering efforts of PUI faculty who had founded the Council on Undergraduate Research (CUR) were beginning to validate the power of undergraduate research in sparking students' passion for becoming research or academic scientists among many other benefits.

Many faculty in the early days of PKAL were also CUR leaders. Together with a diverse group of faculty and administrative leaders from PUI campuses across the country, this leadership group set about establishing a vision for undergraduate learning in science and mathematics. This vision was presented to the community at a PKAL National Colloquium at the National Academy of Sciences in 1992. It defined the following as crucial to undergraduate learning in STEM:

- Learning is experiential, hands-on, and steeped in investigation from the very first day.

- Learning is personally meaningful for students and faculty, makes connections to other fields of inquiry, is 
embedded in the context of its own history and rationale, and suggests practical applications related to the experiences of students.

- Learning takes place in a community where faculty are committed equally to undergraduate teaching and to their own intellectual vitality, where faculty see students as partners in learning, where students collaborate with one another and gain confidence that they can succeed. (Project Kaleidoscope 1991)

This vision, however, was in direct contrast to how learning was generally experienced at that time. It had evolved over many months of exploration-studying the work of individuals, departments, and disciplinary communities deeply engaged in improving learning in STEM undergraduate settings.

The need for a kaleidoscopic approach became quickly clear. Attention to spaces had to complement attention to programs and pedagogies.

The challenges of implementing undergraduate research in existing spaces became obvious. Spaces designed for single investigators could not accommodate student-faculty research teams. Neither could they accommodate the contemporary instrumentation and technologies needed by students and faculty to pursue research and advanced study (see, for example, Figure 1, which illustrates Grinnell College's challenges as it sought to make undergraduate research the hallmark of the sciences on its campus). So the essence of undergraduate research as a means to spark the interest of students in a community of science practitioners was dulled.

The PKAL process identified and involved faculty members who were using pedagogical approaches that promoted active learning and experiential learning of students. It also assembled a small cadre of innovative thinkers dedicated to producing spaces that would best serve the students and faculty of the PKAL and CUR communities. For several years, PKAL planned and hosted facilities workshops and consultancies, studied the data from those experiences, and issued its findings (Project Kaleidoscope 1995). Although this 1995 PKAL vision is based on the experience of campuses pursuing better spaces for science, it is a vision, with just a gentle twist, that holds for all fields of learning and research now.

Facilities that work:

- clearly reflect the educational goals for the sciences and mathematics within an overall institutional framework, for the immediate and the long term.

- support learning that is experiential, hands-on.

- recognize the increasingly social character of scientific research and teaching by facilitating productive interaction between and among students and faculty.
FIGURE 1. PKAL Archive Photo of a Chemical Fume Hood at Grinnell College Reflecting a Need to Modernize Science Facilities (photo courtesy of Jeanne L. Narum)

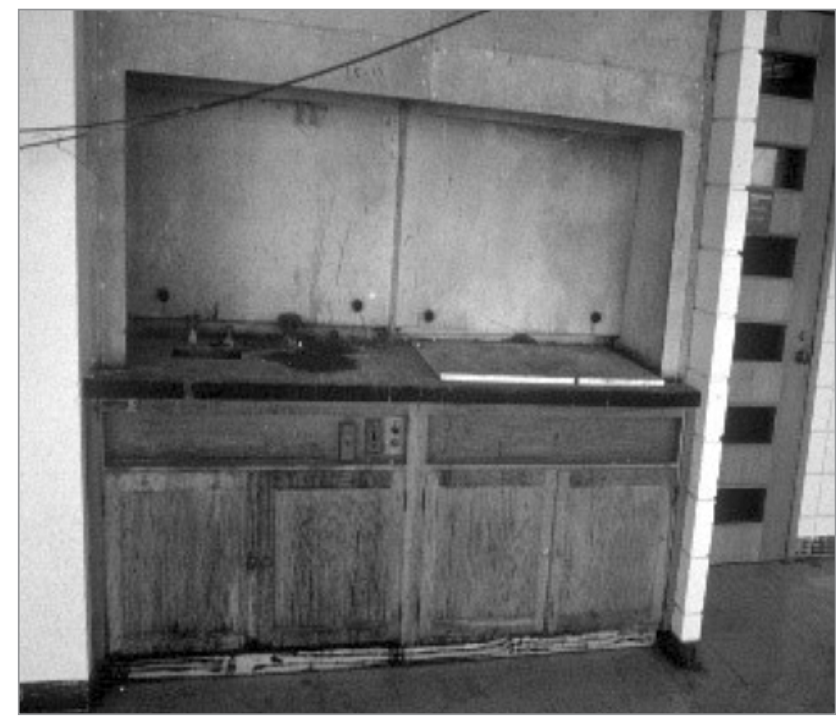

- acknowledge the role of serendipity in the doing of science by including spaces for exploiting the unplanned, the teachable moment.

- are so inviting, safe, and well-equipped that they are used by students and faculty most hours of the day, seven days a week.

- anticipate the future by providing flexibility in space and infrastructure.

- respect and reflect the community that brought them into being.

- contribute to the humanity of the campus. (Project Kaleidoscope 1995)

The following examples from Monmouth University and Skidmore College illustrate the process of conceiving, designing, and implementing learning spaces in science for the twenty-first century.

\section{Monmouth University: A Prototype-Demonstration Approach for Consensus Building}

Reflective of science buildings from the Sputnik era, Monmouth University (MU) spaces were designed for a different time and style of teaching. The importance of facilities to support active learning approaches or facultystudent interactions such as undergraduate research had not been considered in the design. Further, when university status was granted in 1995, the institution had embraced a teacher-scholar model for faculty development with undergraduate research as a core tenet. Existing spaces were a barrier; insufficient to accommodate increasing needs to support this emphasis on undergraduate research and faculty scholarship. The repurposing of former larger lecture spaces, offices, computer labs, and even restrooms over many years were patchwork attempts 
to accommodate student-faculty research labs, smaller classrooms that were more flexible, and student study spaces, resulting in a fragmented, confusing layout.

Across the institution, there was recognition that science facilities needed to be addressed, not only because the science building was the oldest on campus but also because a fundamental lack of understanding existed about modern building designs for modern science and the best way to proceed. Early thinking regarding the project produced a budget limit of $\$ 10$ million. From the planning team perspective, it was quickly recognized that an institutional culture and consensus needed to be developed, promoting a new approach to intentional design of facilities that engaged all stakeholders - from students and faculty to the university president, the board of trustees, and senior leaders responsible for academics, finance, buildings and grounds, and advancement. The following outlines MU's two-phase process of consensus building.

\section{Phase 1: Preparing to Make the Case}

A Facilities Committee was formed, which was led by Michael A. Palladino, then dean of the School of Science. This team attended a PKAL Facilities Workshop, as well as visited and made comparative analyses of science facilities at institutions in New Jersey, New York, and Pennsylvania. Student roundtables were organized to seek input about facility design from the most important users of the spaces. A campus planning firm was contracted to undertake the process of engaging all stakeholders; current spaces were inventoried and future spaces were identified that could reflect the teacher-scholar model, including an emphasis on student-faculty research. A vision for the future was developed that made the conduct of science visible and thus put "science on display." Phase 1 lasted three years.

\section{Phase 2: Building Consensus}

Conversations with stakeholders continued, engaging staff members, trustees, external advisory committees, and councils. A consensus on the vision for the project was achieved, and the Facilities Committee received approval to present a request for proposals to the design community. The committee, working with the architect, first designed a phased plan for an expanded and completely renovated building. The building was designed with a series of integrated modules that included a modern teaching laboratory, student-faculty research laboratory suites, student study spaces, and faculty offices (see Figure 2). These modules would be repeated throughout the entire building. This approach served the goals of increasing human interaction, providing desired proximity of research and classroom teaching spaces, and maintaining activity throughout the building. Through a comprehensive, iterative process of meetings, consensus continued to build, which led to an institutional commitment to
FIGURE 2. Rendering of the Prototype Space (2A) at Monmouth University and Student Occupying New Designed Prototype Space (2B) Prior to Issuance of Certificate of Occupancy (photos courtesy of Michael A. Palladino)

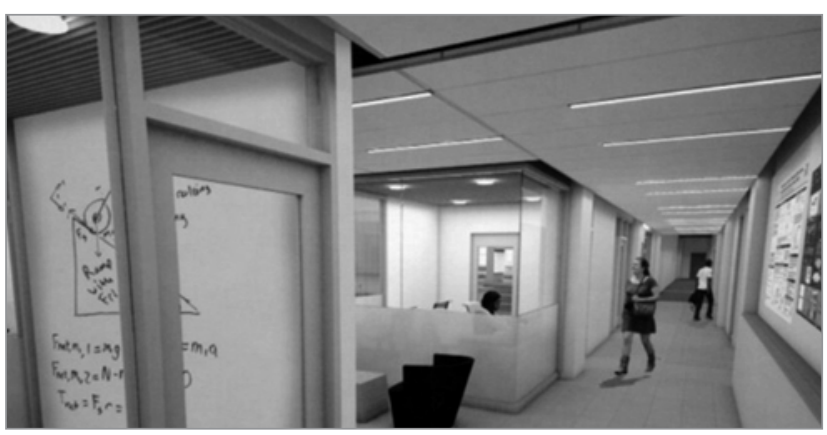

2A

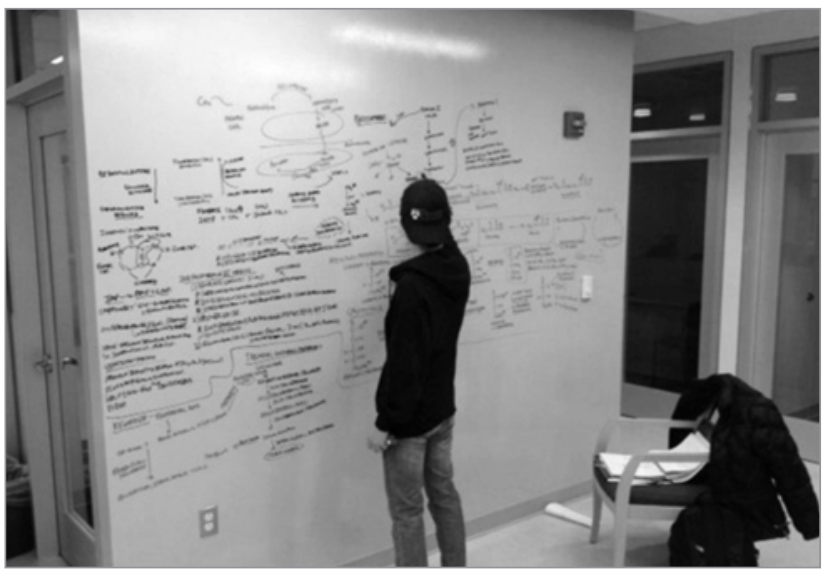

2B

provide $\$ 1.2$ million for the development of a complete pilot module in approximately 25 percent of previously renovated space on one floor.

This prototype was a highly successful, visually compelling "demonstration" of what the new building could be. It revealed how old facilities could be turned into an unrecognizable new and modern space. It was a stark contrast to spaces previously used. For academics inexperienced with spatial design, this was a real-life showcase of why spaces matter. It brought one-dimensional building renderings to life, presenting tangible evidence of what could be delivered by the architectural team, as well as of the power of the vision shaped by the Facilities Committee. The students began to own the space even before the formal Certificate of Occupancy was issued.

The prototype immediately galvanized support for the entire proposed project. The board of trustees made this its highest priority for facilities, committing to a $\$ 48$ million renovation and expansion project. The science building was the largest capital project for an academic building on campus, signaling a commitment to, and 
importance of, the life and physical sciences for the university's students and faculty. The building was completed in December 2017.

\section{Skidmore College: Prioritizing Function to Increase Efficiency and Decrease Cost}

Like many peer institutions, the sciences at Skidmore College suffered from its own success. The sciences had grown into and out of available spaces dispersed among five buildings across campus. Much like MU, an initial planning effort identified needs that would exceed $\$ 150$ million-more than the college could afford. But, at Skidmore, "Creative Thought Matters," so a new approach to facilities planning was sought that would be more efficient while addressing most, if not all, of the campus-wide needs identified. It was recognized that this would only be possible if interdisciplinary science was considered in a twenty-first-century way that went against the ingrained culture and challenged faculty to stretch to new ways of thinking. The plan: to create a Center for Integrated Sciences (CIS).

Several early steps were taken to ascertain what was already happening on campus. A priority was to look for spaces identified as interdisciplinary-spaces that challenged students to learn in different ways and take responsibility for their own learning. Skidmore's Frances Young Tang Teaching Museum and Art Gallery offered an inhouse model to inform the planning process. The "Tang," with its open and flexible design, facilitates object-based instruction in every discipline taught at Skidmore. Rather than looking to replace spaces one for one, stakeholders (students, faculty, staff, and administrators) were interviewed about the activities they would want to accomplish in new space. Campus representatives attended a workshop hosted by the Learning Spaces Collaboratory (LSC) that helped define the right questions to ask during these discussions.

With the assistance of the design team, information was collected about possible shared methodologies in teaching and research as well as about possibilities for shared technologies and major research equipment. These were challenging discussions as they involved all 70 tenuretrack faculty in the 10 STEM programs and forced faculty to transition from a model where individuals "owned" spaces to a shared model. However, by focusing on the activities in each space, initial resistance was overcome. This process also led to thinking about interdisciplinarity and student learning at every level.

By focusing on the activities to be performed in spaces, traditional disciplinary silos could be broken down, and organic affinities could be built that enabled participants to think in new and different ways about integrating the sciences at Skidmore. As one example, molecular life scientists in biology, chemistry, and neuroscience had common methodological approaches and infrastructure needs. They designed shared teaching and research spaces that met all of their needs in less space and with more flexibility.

Activity-first discussions also guided the design of individual clusters of research and teaching spaces. One example of teaching spaces involves a cluster of rooms-a flexible teaching lab, a preparation space, and an auxiliary work ("come-back") space. Authentic scientific investigations do not fit neatly into 3-hour scheduled blocks, but dedicating a lab for longer periods of time worked against the goal of efficiency. The smaller auxiliary work areas provide flexibility to engage students in the research activities documented by CUR (2012) as more effective for student learning while providing opportunities for student mentoring and cross-disciplinary discussion. These measures did not result in an increase in the number of teaching labs needed in the CIS.

After these explorations and dialogues between the 90 members of the science faculty and architects, designers, technologists, and faculty in nonscience disciplines, a final design was achieved from an unprecedented act of collaborative ingenuity. The design represented a melding of cross-disciplinary aspirations for an integrated experience in learning and research for students. It also is a judicious and responsible use of college resources. By starting with questions about activities - about how the spaces would be used by faculty and students alike-a new paradigm could be envisioned for learning at Skidmore.

\section{Next Steps}

The LSC was established in 2010 to build on more than 20 years of attention to the physical environment for undergraduate learning under the PKAL umbrella. As the examples from Monmouth University and Skidmore College indicate, what is important is engaging a diverse community of stakeholders, asking provocative questions, understanding the institutional culture, and having ambitious goals. The strategy of learner-centered planning drove each of those projects.

The LSC began by looking for pioneering faculty who had been approaching space planning over the past decade with a vision for future uses of campus spaces (Learning Spaces Collaboratory 2013). Accounts from 19 colleges and universities were analyzed and compiled into a resource for the community - a guide for learner-centered planning (Learning Spaces Collaboratory 2013). Some institutions described what their students would be doing in these spaces:

... [students would be t]ackling ill-structured, open-ended complex problems with others[;] [s]earching for, finding, 
and sharing relevant, reliable, and up-to-date data with team members $[;]$. . . [t]rying, failing, recovering.

-Problem-Driven Learning Spaces,

Georgia Institute of Technology

(Learning Spaces Collaboratory 2013, 42)

... [students would be] collaborating with peers on interesting tasks, [in...] "hands-on" engagement with solving content-rich problems[;] [p]resenting and evaluating oral arguments; viewing and critiquing the work of individual teams.

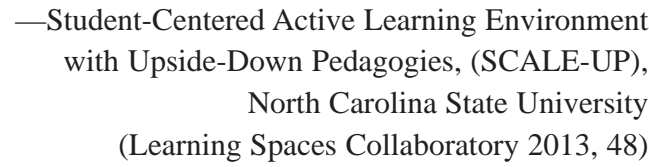

... [students would be] feeling comfortable in an open, accepting work and classroom environment that encourages experimentation and risk taking. ... [e]njoying a sense of physical freedom, with the ability to get up, move around, join others, demonstrate ideas.

-Michelle Smith Collaboratory for Visual Culture, University of Maryland-College Park

(Learning Spaces Collaboratory 2013, 58)

The accounts from these institutions highlighted how research findings in cognitive science and the field are influencing those responsible for learning environments in the twenty-first century. There is a growing national awareness that space matters to learning. At the institutional level, there is a deeper understanding of the complexity and consuming nature of the planning process for learning spaces. The character and scope of questions now being asked is the most significant mark of how planning of learning spaces has changed over the past decade.

The following questions drove the planning of the Watt Center for Student Services at Clemson University (Learning Spaces Collaboratory 2017; see figure 3):

- How do we create a campus-wide "heart" for student engagement and innovation?

- What is our mental image of an agile, timeless environment for learning and research?

- How do we create a destination for learning that fosters collaborations beyond the campus that help students build a bridge to the future?

The Clemson account is included in portfolios from LSC roundtables focusing on the future of planning learning spaces. These roundtables were designed as a strategy adapted from the beginning of PKAL: gather a diverse group of reflective practitioners, challenge them to point the way, and translate their insights into roadmaps for the LSC community.

The roundtables identified audacious questions that have implications for the planning and use of space. As the LSC
FIGURE 3. Watt Center for Student Services at Clemson University (photo courtesy of Jeanne L. Narum)

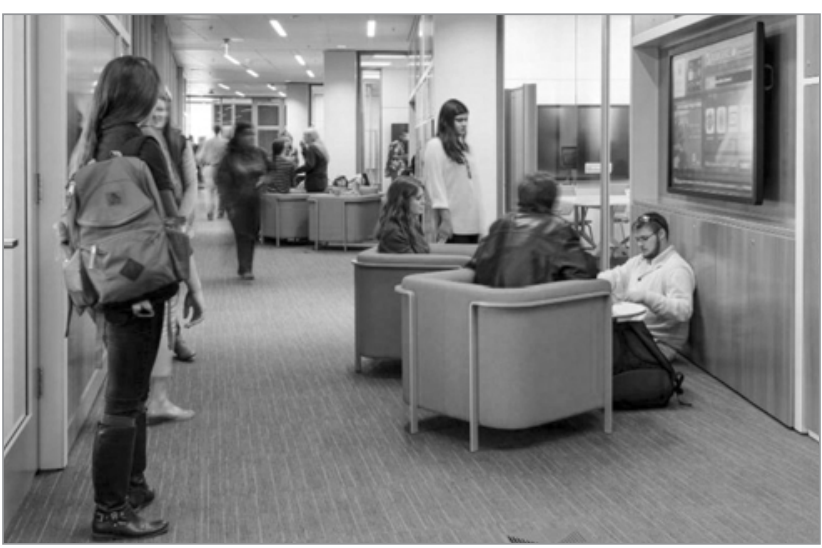

and CUR communities ask "what next," the following questions from the roundtables can serve as one guide into the future of planning learning spaces:

- How do we help form the relationships, environments, and events that are mind- and heart-altering, that challenge, refresh and inspire how learning happens?

- Students need community. We all do. How can spaces define and encourage different senses of community?

- If we build it, will they come?

- How do we create those environments that define what the institution is about?

- How can the institutional context-geographical and cultural-influence the planning of a facility?

- How do we avoid having the building and its spaces define how we would like to teach and learn?

- What do we want learners to become, to be able to do when they leave campus? (Learning Spaces Collaboratory 2013).

The final question points to questions about the world beyond the campus, questions about the changing context and how it influences planning. As before, national reports suggest new questions and possible solutions as the CUR community, together with the LSC and others across the country explore answers to the what next question. A working group of the National Research Council is pursuing the following question: should STEM become $A+$ $H+S T E M+M$, with the arts, humanities, and medicine embracing STEM? Some campuses are already addressing this question indirectly. Like the questions about undergraduate research so many years ago, it is a question that will influence the planning of learning spaces in the future.

\section{References}

Council on Undergraduate Research. 2012. Characteristics of Excellence of Undergraduate Research (COEUR). Washington, DC: Author.

Learning Spaces Collaboratory. 2013. "A Guide Planning for Assessing 21st Century Spaces for 21st Century Learners." 
Accessed September 4, 2017. http://www.pkallsc.org/assets/files/ LSCGuide-PlanningforAssessing(1).pdf

Learning Spaces Collaboratory. 2017 “An LSC Webinar: Learning Spaces Matter-Academics and Architects: The Conversation Continues." Accessed December 8, 2017. http://www. pkallsc.org/basic-page/lsc-webinar-learning-spaces-matter-academics-and-architects-\%E2\%80\%8B-conversation-continues

National Science Board (NSB). 1986. Undergraduate Science, Mathematics and Engineering Education: Role for the National Science Foundation and Recommendations for Actions by Other Sectors to Strengthen Collegiate Education and Pursue Excellence in the Next Generation of U.S. Leadership in Science and Technology. Washington, DC: NSB Publications. Accessed September 4, 2017. https://www.nsf.gov/nsb/publications/1986/ nsb0386.pdf

Project Kaleidoscope. 1991. "Volume I: What Works: Building Natural Science Communities." Accessed September 4, 2017. http://www.pkal.org/collections/WhatWorks.cfm

Project Kaleidoscope. 1995. "Volume III: Structures for Science."
Accessed September 4, 2017. http://www.pkal.org/collections/ VolumeIII.cfm

\section{Kimberley Frederick}

Skidmore College, kfreder1@skidmore.edu

Jeanne L. Narum is principal of the Learning Spaces Collaboratory (LSC) in Washington, DC. From 1989 to 2010, Narum was director of Project Kaleidoscope (PKAL), an NSF-funded initiative to catalyze transformation of undergraduate learning environments in STEM.

Kimberley Frederick is professor of chemistry at Skidmore College. Her research group focuses on developing new microfluidic technologies to perform chemical analyses.

Michael A. Palladino is vice provost for graduate studies and professor of biology at Monmouth University. His research includes antimicrobial properties of male reproductive organs, as well as molecular mechanisms of oxygen homeostasis of the testis. 